\title{
Demographics and Population Dynamics Project the Future of Hard Coral Assemblages in Little Cayman
}

\author{
Kristi A. Foster, Greg Foster \\ Coastal \& Marine Ecology Consultants, Inc., Nehalem, OR, USA \\ Email:kfoster@coastalmarineecology.com, gfoster@CoastalMarineEcology.com
}

How to cite this paper: Foster, K.A. and Foster, G. (2018) Demographics and Population Dynamics Project the Future of Hard Coral Assemblages in Little Cayman. Open Journal of Marine Science, 8, 196-213. https://doi.org/10.4236/ojms.2018.81010

Received: December 26, 2017

Accepted: January 28, 2018

Published: January 31, 2018

Copyright $\odot 2018$ by authors and Scientific Research Publishing Inc. This work is licensed under the Creative Commons Attribution International License (CC BY 4.0).

http://creativecommons.org/licenses/by/4.0/

\begin{abstract}
Individual hard coral colonies from four representative reef sites around Little Cayman were surveyed yearly between 2010 and 2015, a period of non-disturbance between two elevated seawater temperature anomalies. Photographic censuses produced 7069 annual transitions that were used to describe the demographics (size class frequencies, abundance, area cover) and population dynamics under non-disturbance environmental conditions. Agariciids, Porites asteroides, and Siderastrea radians have replaced acroporids as the predominant massive corals. Recruitment rates were generally low $(<1$ colony per $\mathrm{m}^{2}$ ), except for a fourfold recruitment pulse of $S$. radians that occurred in 2011. On average, $42 \%$ of coral recruits survived their first year but only $10 \%$ lived longer than four years. Temporal comparisons allowed correction factors to be calculated for in-situ methods that overestimate recruitment of colonies $\leq 2 \mathrm{~cm}$ in diameter and overlook larger colonies. Size class transitions included growth $(\sim 33 \%)$, stasis $(\sim 33 \%)$, partial mortality $(10 \%-33 \%)$, and whole colony mortality, which decreased with increasing colony size (typically $<10 \%$ for colonies with surface areas $>30 \mathrm{~cm}^{2}$ ). Transition matrices indicated that Little Cayman assemblages have declining hard coral populations $(\lambda<1)$ but as stable size class distributions progress toward higher proportions of colonies with $>150 \mathrm{~cm}^{2}$ surface areas, live area cover may remain relatively stable. Projection models indicated that downward population trends would be exacerbated even by mild disturbance ( $5 \%-10 \%$ mortality) scenarios. The fate of hard corals on Little Cayman's reefs was determined to be heavily dependent on the health and transitions of agariciid colonies. Conservation strategies that currently focus on restoration of Caribbean acroporids should be expanded to include agariciids, which were previously considered "weeds".
\end{abstract}

\section{Keywords}

Caribbean Coral, Size Class Transitions, Vital Rates, Recovery Projections, 
Little Cayman

\section{Introduction}

Since the 1970s, Caribbean corals have been subjected to regional and local mass mortality events associated with disease outbreaks, temperature-induced bleaching, hurricanes, and anthropogenic stressors, which have resulted in a decline in average coral cover from $35 \%$ to $16 \%$ [1]. The previously dominant reef builders, Acropora cervicornis and A. palmata, have been disproportionately susceptible to these disturbances and have been placed on the IUCN's Red List as critically endangered. Coral dominance has shifted from these highly susceptible branching and tabular species to more resistant and resilient massive taxa. The fates of the surviving massive corals shall shape future Caribbean coral communities.

Little Cayman is an ideal location within the Caribbean to study the population dynamics of hard coral populations because it lacks many of the anthropogenic stressors to which other reefs are exposed. Little Cayman is located $120 \mathrm{~km}$ northeast of Grand Cayman, $145 \mathrm{~km}$ south of Cuba, and $10 \mathrm{~km}$ southwest of Cayman Brac (Figure 1). The remote island is approximately $16 \mathrm{~km}$ long by 1.6 $\mathrm{km}$ wide, with a maximum elevation of $12 \mathrm{~m}$, and is surrounded by shallow and narrow reef shelves, some of which drop off to vertical walls (e.g. Bloody Bay Wall) that reach $300 \mathrm{~m}$ deep. More than half of the shallow reefs have been designated as protected areas (e.g. marine parks, replenishment zones) since 1986 [2].

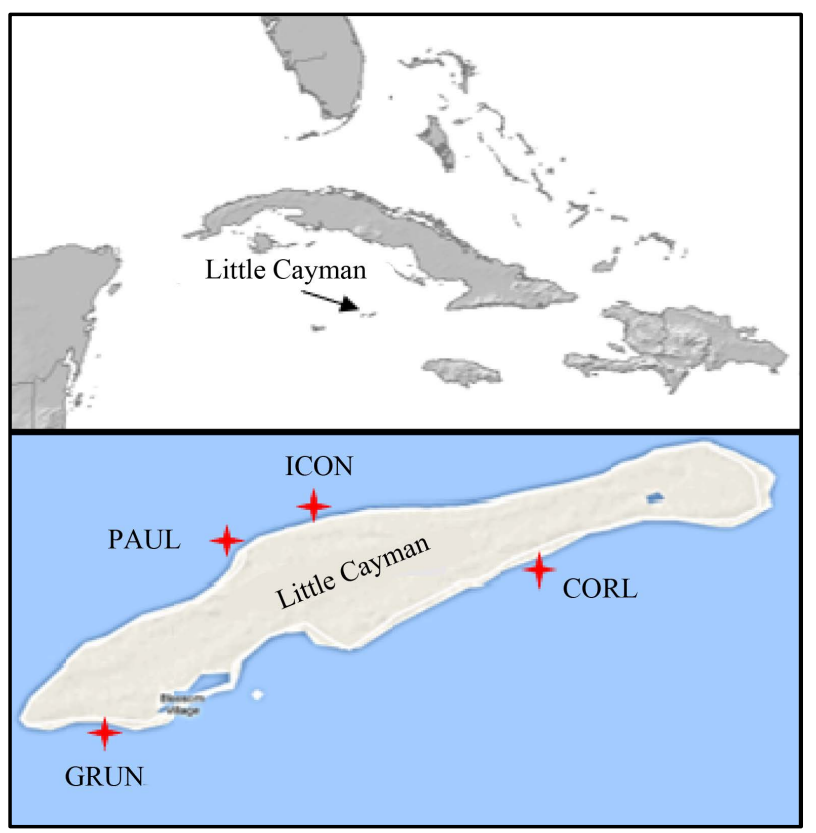

Figure 1. Map of Little Cayman study areas. Monitoring station locations at Coral City (CORL), Grundy's Garden (GRUN), ICON Reef (ICON), and Paul's Anchors (PAUL). 
Little Cayman has a small resident population ( $<200$ people) and lacks agriculture, freshwater input rivers or streams, commercial fishing, industry, and ports; providing a rare opportunity to decouple the impacts that anthropogenic stressors (e.g. nutrient run off, sedimentation, pesticides, pollution, resource extraction, development) and environmental stressors have on the health of coral reefs.

Live coral cover in Little Cayman has rebounded from its low point of $14 \%$ in 2004 to an average of $25 \%$, among the highest in the Caribbean, despite sharing a similar disturbance history with reefs region wide [1] [3] [4]. Little Cayman corals experienced elevated temperature-induced bleaching and mortalities of various intensities during 1987, 1995, 1998, 2003, 2005, 2009, and 2015. The island experienced direct hits by hurricanes in 1980 (Allen, category 4), 1988 (Gilbert, category 4), and 2008 (Paloma and Gustav, categories 4 and 2, respectively). Outbreaks of white, yellow, and black band diseases and white plague have followed hurricane and bleaching events in 1981-82, 1990, 1996, and 1999-2000.

The study reported herein took place annually from 2010 through 2015 during non-disturbance environmental conditions (i.e. between the two most recent elevated temperature anomalies and during a period when no hurricanes, disease outbreaks, or other mortality events occurred). The objectives of this study were to 1) describe the demographics and dynamics of the hard coral communities around Little Cayman, 2) use the vital rates, based on temporal comparisons of individual colonies, to develop size class transition probability matrices, and 3) project the recovery potential of these coral assemblages for several disturbances scenarios.

\section{Methods}

\subsection{Annual Surveys}

Scleractinian coral populations were surveyed annually along the reef crests at four locations around Little Cayman for six consecutive years, between 2010 and 2015, during the non-disturbance period between two elevated seawater temperature anomalies (i.e. annual surveys began one year after the 2009 bleaching event and ended two months prior to first signs of coral paling in 2015) (Figure 1, Table 1). Permanent monitoring stations were installed, with permission from the Cayman Islands Department of Environment, in order to allow for repetitive photographic surveys of benthic areas and specific coral colonies. Digital images were taken each summer along three $10 \mathrm{~m} \times 1.5 \mathrm{~m}$ belt transects radiating from a central point within each monitoring station at depths of $9-15 \mathrm{~m}$ using a rigid photo-framer that oriented the camera at a fixed distance of $50 \mathrm{~cm}$ above the benthos. The $0.5 \mathrm{~m} \times 0.75 \mathrm{~m}$ base of the framer served as a border within each image to provide known dimensions for subsequent image analysis. Monitoring stations were haphazardly located to avoid crossing over sand channels which are unsuitable for scleractinian settlement. 
Table 1. Descriptions of repetitive monitoring sites.

\begin{tabular}{cccccc}
\hline Station & Site Name & Depth $(\mathrm{m})$ & Latitude & Longitude & MPA \\
\hline CORL & Coral City & $11-13$ & N19.68075 & W80.02330 & No \\
GRUN & Grundy's Garden & $9-12$ & N19.65733 & W80.08955 & Yes \\
ICON & ICON Reef & $11-12$ & N19.69960 & W80.06058 & Yes \\
PAUL & Paul's Anchors & $11-15$ & N19.69443 & W80.06943 & Yes \\
\hline
\end{tabular}

MPA = Marine Protection Area.

\subsection{Image Analysis}

Each scleractinian coral which appeared as a whole colony within a given belt transect was traced using the Area Analysis function in Coral Point Count (CPCe) [5], which calculated colony area cover (planar view). Image resolution was clear enough to identify colonies as small as $0.1 \mathrm{~cm}^{2}$ to species; however, some of the smaller Agaricia, Undaria, and Helioseris spp. colonies lacked the morphological characteristics that help to differentiate the species, so these taxa were pooled into the "agariciid" group. Colonies which first appeared within a given transect after the initial 2010 survey were considered recruits. Colonies were presumed to have died if they were absent after the initial survey, overgrown by Trididemnum solida tunicates or macroalgae, or no longer had live tissue visible on the skeletons.

\subsection{Size Class Determination}

Hard corals were grouped into eight size-dependent classifications (“SC") (Table 2) based on area cover. To determine the most appropriate groupings, size classifications were compared for areas associated with radius increments of $1 \mathrm{~cm}$ and 2 $\mathrm{cm}$, assuming colonies closely resembled circular structures with Area $=\pi \mathrm{r}^{2}$. Each of the top 10 most abundant taxa was distributed across either size class grouping, except for Siderastrea radians, the third most abundant taxa. If the larger size classes (based on $2 \mathrm{~cm}$ radius increments) were used, $>97 \%$ of all

Table 2. Size-dependent classifications for hard coral colonies.

\begin{tabular}{ccc}
\hline Size Class & Area Cover $\left(\mathrm{cm}^{2}\right)$ & Approx Radius $(\mathrm{cm})$ \\
\hline SC1 & $<4$ & $<1$ \\
SC2 & $4-12$ & 2 \\
SC3 & $12-30$ & 3 \\
SC4 & $30-50$ & 4 \\
SC5 & $50-75$ & 5 \\
SC6 & $75-100$ & 6 \\
SC7 & $100-150$ & 7 \\
SC8 & $>150$ & $>8$ \\
\hline
\end{tabular}

Hard coral colonies were grouped into eight size classes based on their measured area cover and estimated radii (assuming circular colonies, $\mathrm{A}=\pi \mathrm{r}^{2}$ ). $\mathrm{SC}=$ size class. 
S. radians colonies would be combined into the smallest size class (SC1) and their growth and partial mortality rates would be masked as size class stability which could, in turn, mask the overall vitality rates of Little Cayman's corals. Therefore, size classes based on $1 \mathrm{~cm}$ radius increments were used for the analysis described herein.

\subsection{Transition Matrices}

Size class transition matrices were developed for the two most abundant taxa (agariciids and Porites asteroides) and for all hard corals pooled together to represent Little Cayman overall. ( $S$. radians, the third most abundant taxa, was comprised of small colonies in SC1-3 only; therefore, the respective size class transitions were not developed separately.) The use of eight size classes resulted in $8 \times 8$ matrices in which each element represents the mean probability of moving from a starting size class or "state" (column) to ending size class or "fate" (row) [6] [7]. The matrices include growth (G) to the next largest size class, size class stability (S) by remaining within the same group, or partial mortality (PM) to a smaller size class. Corals may also experience fission (i.e. the regression of a single colony into multiple smaller ramets) or fusion (i.e. two or more ramets grow together) [8] [9]. In these cases, which were rare in this study, the area cover for all ramets were pooled and compared to the size class for the respective parent colony which underwent fission or for the resulting fused colony and were recorded as a partial morality, size class stability or growth transition. The resulting probability matrices were used to project the number of corals in each size class during year $t+1$, which equals the number in each size class at year $t$ multiplied by the respective size class transition probabilities plus the mean number of corals which enter the population through recruitment (R) (Equation (1)).

$$
\left.F(n)=\left(\begin{array}{l}
S C 1 \\
S C 2 \\
S C 3 \\
S C 4 \\
S C 5 \\
S C 6 \\
S C 7 \\
S C 8
\end{array}\right)_{(t+1)}=\left\{\left[\begin{array}{cccccccc}
S & P M & P M & P M & P M & P M & P M & P M \\
G & S & P M & P M & P M & P M & P M & P M \\
G & G & S & P M & P M & P M & P M & P M \\
G & G & G & S & P M & P M & P M & P M \\
G & G & G & G & S & P M & P M & P M \\
G & G & G & G & G & S & P M & P M \\
G & G & G & G & G & G & S & P M \\
G & G & G & G & G & G & G & S
\end{array}\right] \times\left(\begin{array}{c}
S C 1 \\
S C 2 \\
S C 3 \\
S C 4 \\
S C 5 \\
S C 6 \\
S C 7 \\
S C 8
\end{array}\right)_{(t)}\right)\right\}_{R}\left(\begin{array}{c}
R 1 \\
R 2 \\
R 3 \\
R 4 \\
R 5 \\
R 6 \\
R 7 \\
R 8
\end{array}\right)
$$

An $8 \times 8$ matrix has eight eigenvalues, $\lambda_{\mathrm{i}}$, or solutions to the matrix. The dominant eigenvalue (i.e. the largest, positive eigenvalues that is a real number) is the growth rate of the size class-structure population [6] [10]: 1) for $\lambda>1$, the population is growing, 2) for $\lambda=1$, the population is stable, and 3) for $\lambda<1$, the population is declining. The ratio of the dominant eigenvalue to the absolute value of the second largest eigenvalue, known as the damping ratio, provides the 
rate of convergence of the population toward a stable stage distribution (i.e. the larger the damping ratio, the quicker a population will return to its stable state after a disturbance) [6] [7]. Sensitivities and elasticities are measures of perturbation analyses that quantify the relative contribution of each vital rate to the population growth by adjusting each rate by a specific amount and by a specific proportion, respectively [6] [7]. The dominant eigenvalues (i.e. population growth rates), stable size class distributions, sensitivities, and elasticities for the transition matrices were calculated using the PopTools add-in for Excel [11].

\subsection{Projection Models}

Projections were modeled through 2040 as idealized, best-case scenario forecasts of massive coral populations [6] [7]. Such projections assumed that 1) current environmental parameters remain unchanged over time, (i.e. during disturbance-free intervals); 2) coral vital rates (e.g. growth, stability, partial mortality) include the interactions among corals and other benthic organisms, responses to the surrounding environment, and other factors that affect population structures; 3 ) mean colony area in each size class remains unchanged over time; and 4) the mean recruitment rates between 2010-2015 occur annually throughout the projection period.

\section{Results}

\subsection{Demographics within Monitoring Stations}

A total of 7069 annual transitions were recorded during the six-year study. The sample population was comprised of 23 taxa groups (Figure 2, Table 3). Additional species (e.g. A. cervicornis, A. palmata, P. divaricata, P. porites) are

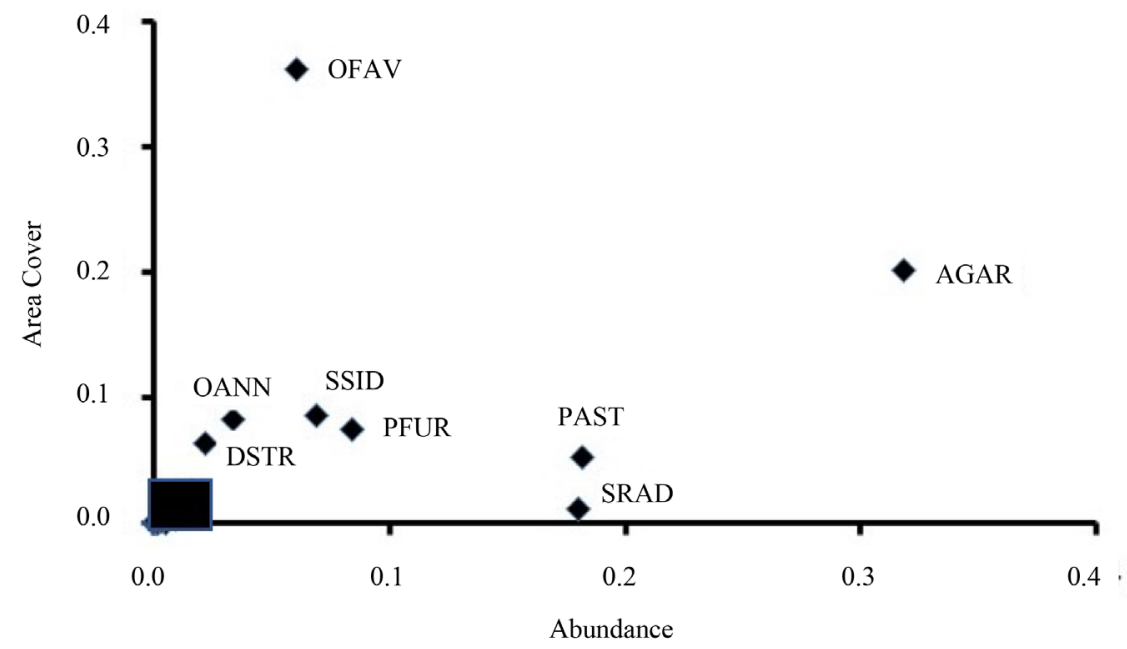

Figure 2. Fractional abundance and area cover for hard coral taxa at monitoring stations. $\mathrm{AGAR}=$ agariciids; $\mathrm{PAST}=$ Porites asteroides $\mathrm{SRAD}=$ Siderastrea radians, $\mathrm{PFUR}=P$. furcata; $\mathrm{SSID}=S$. siderea , OFAV $=$ Orbicella faveolata , OANN $=$ O. annularis, $\mathrm{DSTR}=$ Diploria strigosa. Black rectangle at axis indicates positions of the other 15 taxa inventoried. 
Table 3. Hard coral taxa within monitoring stations.

\begin{tabular}{ccc}
\hline Abundant & Common & Rare \\
\hline Agariciids & Diploria strigosa & Dendrogyra cylindricus \\
Porites asteroides & D. labrynthiformis & Eusmilia fastigiata \\
Siderastrea radians & Dichocoenia stokesi & Favia fragum \\
P. furcata & Montastrea cavernosa & Isophyllia sinuosa \\
S. siderea & Mycetophyllia spp. & Madracis dectactis \\
Orbicella faveolata & Orbicella annularis & Manicina areolata \\
& O. franksii & Meandrina spp. \\
& Stephanocoenia intercepta & Solenastrea bournoni \\
& & Scolymia spp. \\
\hline
\end{tabular}

Abundant $=$ Greater than 100 individual colonies recorded within the belt transects during one or more years; Common $=$ Between 5 and 100 colonies; Rare $=$ Less than 5 colonies.

present, and may be prevalent in certain areas around the island, but were not recorded within the belt transects.

Agariciids, Porites asteroides, and Siderastrea radians collectively comprised $>68 \%$ of the hard coral communities.

$70 \%$ of all colonies within the study area were smaller than $30 \mathrm{~cm}^{2}$ (SC1-SC3). Each of the larger size classes (S4-S8) contributed less than 10\% to the total population each year (Figure 3). The largest temporal size class fluctuation occurred in the smallest (SC1) corals, primarily due to a recruitment pulse of $S$. radians in 2011 (Figure 4).

\subsection{Recruitment}

Recruits were observed within the monitoring stations for all recorded taxa except D. cylindricus, D. labrynthiformis, F. fragum, I. sinosa, and Meandrina spp.; however, recruits of these taxa were observed elsewhere around Little Cayman during the study period. A recruitment pulse of $S$. radians occurred in 2011 during which recruit abundance was fourfold greater than during each subsequent year of the study ( 4 recruits per $\mathrm{m}^{2}$ compared to 1 recruit per $\mathrm{m}^{2}$ ) (Figure 4). All other taxa had annual recruitment of $<1$ recruit per $\mathrm{m}^{2}$ between 2011 and 2015. Further investigations are required to identify pulse cycles and possible contributing factors.

Previous studies have estimated coral recruitment in Little Cayman by counting colonies that are $\leq 2 \mathrm{~cm}$ in diameter [4], the equivalent of SC1 in this study. However, such methods significantly overestimate SC1 recruits and overlook recruits in larger size classes. Temporal monitoring indicated that only $60 \% \pm 11 \%$ (five-year mean \pm standard deviation) of the SC1 colonies are recruits; the remaining colonies were visible in prior years and were, therefore, more than one year old. SC2 recruits were common: $35 \% \pm 6 \%$ of SC2 colonies were recruits (Table 4). Among the SC3 colonies, $14 \% \pm 3 \%$ were recruits. The fast growing or 


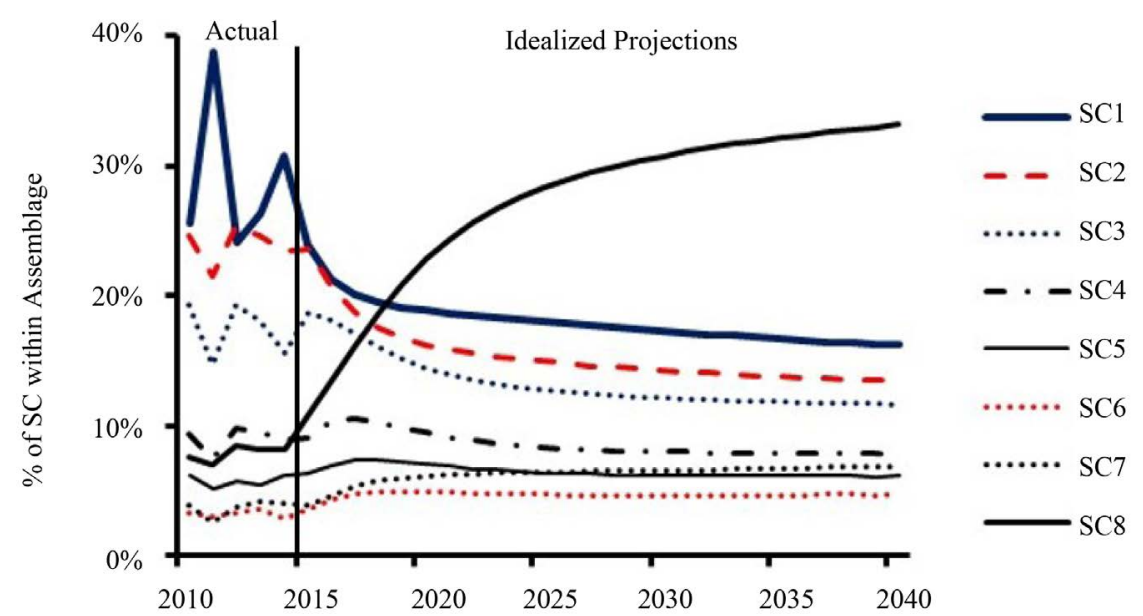

Figure 3. Annual Population Structures for All Hard Corals-Actual and Projected. Idealized projections assume no environmental disturbances or changes in vital rates through 2040. Annual variability is smoothed for idealized projections which are calculated using 5 -year mean probabilities based on the actual population structures. SC $=$ size class with respective area covers as follows: $\mathrm{SC} 1 \leq 4 \mathrm{~cm}^{2}$; SC2 $=4-12 \mathrm{~cm}^{2}$; SC3 $=12-30 \mathrm{~cm}^{2}$; SC4 = $30-50 \mathrm{~cm}^{2}$; SC5 $=50-75 \mathrm{~cm}^{2} ; \mathrm{SC} 6=75-100 \mathrm{~cm}^{2} ; \mathrm{SC} 7=100-150 \mathrm{~cm}^{2} ; \mathrm{SC} 8 \geq 150 \mathrm{~cm}^{2}$.

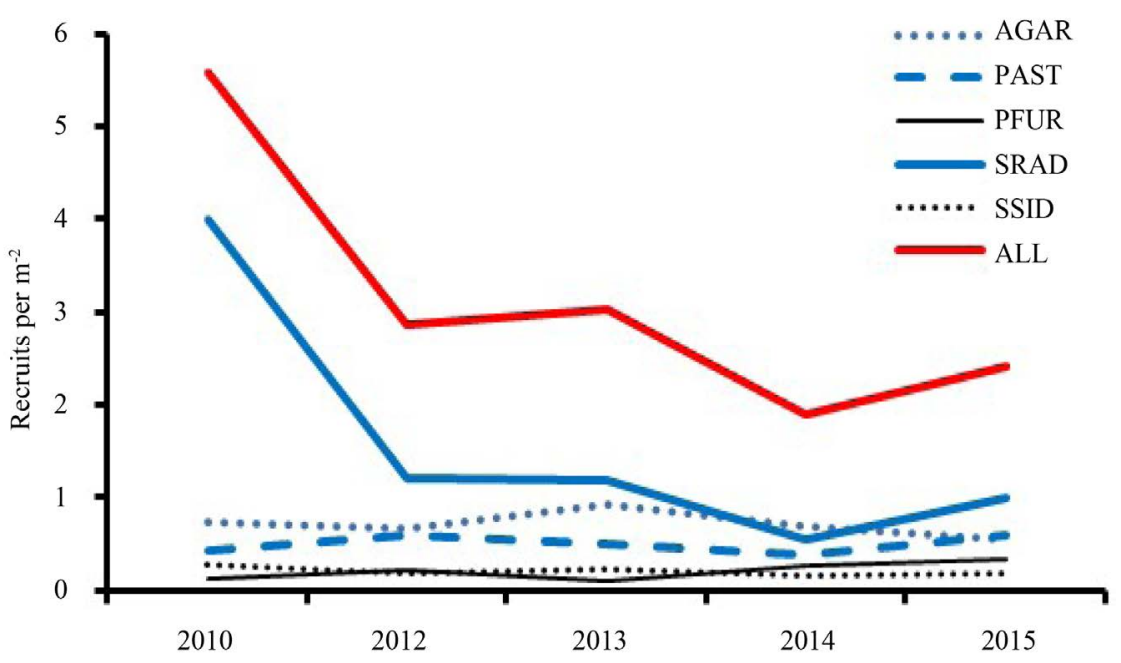

Figure 4. Annual Abundance of Recruits. Top five recruit taxa each year: AGAR = agariciids; $\mathrm{PAST}=P$. asteroides $\mathrm{SRAD}=S$. radians, $\mathrm{SSID}=S$. siderea $\mathrm{ALL}=$ all taxa pooled together.

"weedy" agariciids and $P$. furcata also had recruits as large as SC6 (diameters $\leq$ $12 \mathrm{~cm}$ ): among the SC4-6 agariciid and $P$. furcata colonies, $5 \%( \pm 1 \%)$ and $20 \%$ $( \pm 5 \%)$ were recruits, respectively. No recruits were observed in SC7-8 for any of the taxa. Based on the results of this study, the authors recommend that recruit estimation procedures which use size as a proxy for newly settled corals be modified to 1) count colonies in size classes $1-3$ and apply the correction factors provided in Table 4 for all taxa and 2) include SC4-6 agariciid and P. furcata colonies, adjusted accordingly, among the recruit estimates.

Recruits from all taxa and size classes experienced low survival: $42 \%$ of 
Table 4. Percent recruits within each size class (2011-2015).

\begin{tabular}{ccccc}
\hline Taxa Group & SC1 & SC2 & SC3 & SC4-6 \\
\hline AGAR & $71 \%( \pm 5 \%)$ & $43 \%( \pm 10 \%)$ & $19 \%( \pm 3 \%)$ & $5 \%( \pm 1 \%)$ \\
PAST & $63 \%( \pm 8 \%)$ & $28 \%( \pm 5 \%)$ & $6 \%( \pm 3 \%)$ & $-0-$ \\
PFUR & $68 \%( \pm 19 \%)$ & $59 \%( \pm 27 \%)$ & $41 \%( \pm 14 \%)$ & $20 \%( \pm 5 \%)$ \\
SRAD & $54 \%( \pm 14 \%)$ & $27 \%( \pm 9 \%)$ & $10 \%( \pm 12 \%)$ & $-0-$ \\
SSID & $68 \%( \pm 8 \%)$ & $26 \%( \pm 14 \%)$ & $6 \%( \pm 8 \%)$ & $-0-$ \\
OTHERS & $55 \%( \pm 12 \%)$ & $36 \%( \pm 16 \%)$ & $7 \%( \pm 6 \%)$ & $-0-$ \\
\hline
\end{tabular}

Five-year mean percentages $( \pm$ STD) of colonies within each size class that are recruits. Mean percentages may be used as correction factors for in-situ recruit estimation surveys. AGAR $=$ agariciids; $\mathrm{PAST}=P$. asteroides PFUR $=$ P. furcata; $\mathrm{SRAD}=S$. radians $\mathrm{SSID}=S$. siderea OTHERS $=13$ common and rare taxa of massive corals pooled together.

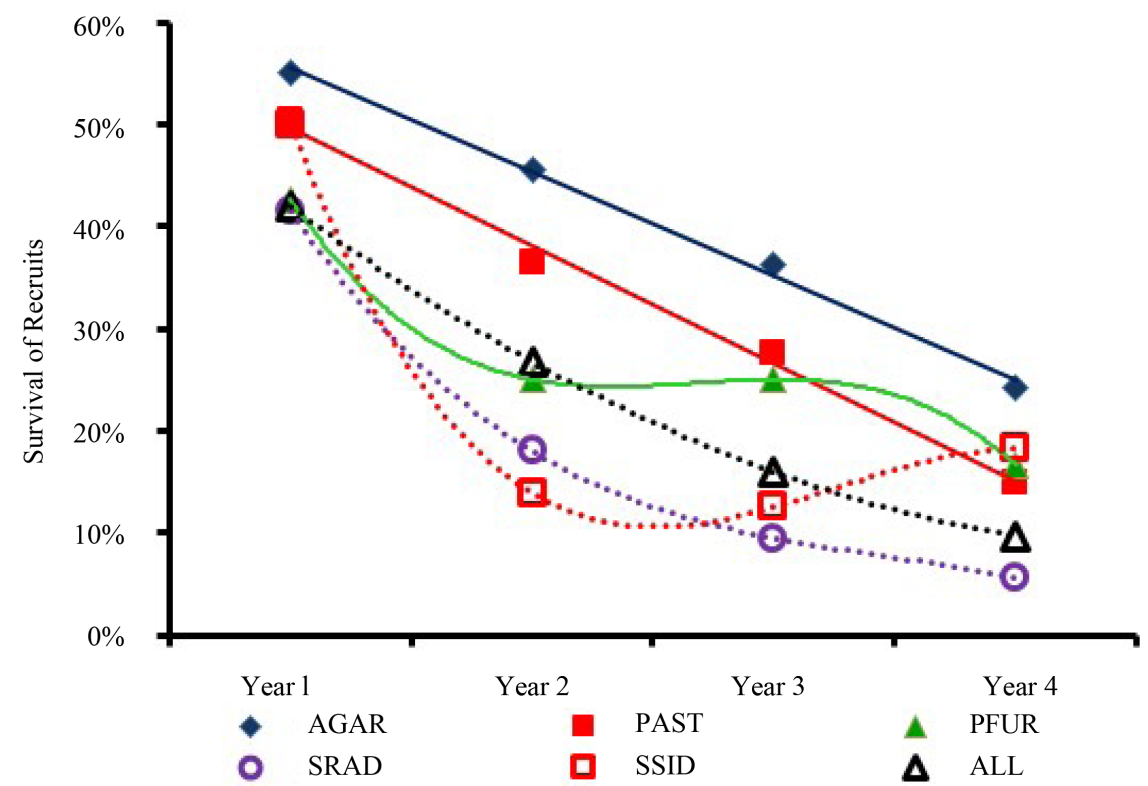

Figure 5. Recruit Survival over Time. Trendline equations are provided in Table 5. $\mathrm{AGAR}=$ agariciids $; \mathrm{PAST}=P$. asteroides $\mathrm{SRAD}=S$. radians $\mathrm{SSID}=S$. siderea $; \mathrm{ALL}=$ all taxa pooled together.

Table 5. Recruit survival rates.

\begin{tabular}{|c|c|c|c|}
\hline $\begin{array}{c}\text { Taxa } \\
\text { Group }\end{array}$ & Trend & Equation & $\mathbf{r}^{2}$ \\
\hline AGAR & Linear & $-0.1016 x+0.6585$ & 0.9962 \\
\hline PAST & Linear & $-0.1151 x+0.6112$ & 0.9939 \\
\hline PFUR & Polynomial & $-0.0433 x^{3}+0.348 x^{2}-0.9175 x+1.0392$ & 1.0000 \\
\hline SRAD & Polynomial & $-0.0172 x^{3}+0.1777 x^{2}-0.6474 x+0.9013$ & 1.0000 \\
\hline SSID & Polynomial & $-0.0461 x^{3}+0.4501 x^{2}-1.3889 x+1.4848$ & 1.0000 \\
\hline ALL & Polynomial & $0.0223 x^{2}-0.2192 x+0.6165$ & 1.0000 \\
\hline
\end{tabular}

Survival trends of recruits. $\mathrm{AGAR}=$ agariciids; $\mathrm{PAST}=$ P. asteroides, $\mathrm{PFUR}=P$. furcata $\mathrm{SRAD}=S$. radians SSID $=S$. siderea $; \mathrm{ALL}=$ all taxa of massive corals pooled together. 
recruits survived their first year; $27 \%$ survived to their second year; $16 \%$ survived to their third year; and $10 \%$ survived to their fourth year (Figure 5, Table 5). Given the high rates of mortality, recruits may die before they reach sexual reproductive maturity; therefore, the authors caution against using recruitment pulses as indicators of reef recovery.

\subsection{Size Class Transitions}

Approximately one-third of the SC1-SC7 colonies grew between monitoring periods regardless of size class (Table 6). Agariciids and $P$. furcata were capable of growing six size classes within a single year, whereas the other taxa groups grew no more than three size classes within the same time frame. However, these large growth spurts were uncommon: $72 \%$ of the growing colonies transitioned to the next larger size class.

Approximately one-third of the SC1-SC7 colonies remained within the same size class. However, $60 \%-100 \%$ of those colonies which achieved surface areas $>150 \mathrm{~cm}^{2}$ (SC8) had size class stability, depending on the taxa group.

Partial mortality varied by size class. Approximately one-third of the SC4-SC7 colonies decreased in size. Among the smaller SC2-SC3 and larger SC8 colonies, $10 \%-15 \%$ experienced partial mortality and transitioned into a smaller size class. Occasionally, shrinkage of four size classes was recorded; however, $74 \%$ of the shrinking colonies transitioned to the next smaller size class.

Between one-third and half of SC1 colonies die each year, largely due to the high mortality of recruits. The probabilities of whole colony mortality decrease with increasing colony size and are typically $<10 \%$ for those colonies with surface areas $>30 \mathrm{~cm}^{2}$ (SC4-SC8). An exception is the apparent increase in mortality for $P$. asteroides, which increases from negligible mortality for SC6-SC7 colonies to $20 \%$ mortality for SC8 colonies. This study was conducted during a period of non-disturbance environmental conditions (e.g. no temperature anomalies, disease outbreaks, extreme storms); therefore, the cause(s) for whole colony mortality require(s) further study.

\subsection{Stable Size Class Distributions, Dominant Eigenvalues, and Damping Ratios}

The stable size class distributions (i.e. the eigenvectors associated with the dominant eigenvalues), dominant eigenvalues and damping ratios were determined for agariciids, $P$. asteroides, and all 23 hard coral taxa pooled together (Table 7). The dominant eigenvalues $(\lambda)$ were $<1$, which result in gradual population decay [6]. The damping ratios were 1.12 - 1.36, indicating the hard corals approach asymptotic behavior (stability) at similar rates among the taxa groups (i.e. similar resilience/recovery rates following a disturbance) [12].

\subsection{Sensitivities and Elasticities}

Sensitivities and elasticities are measures of perturbation analyses that quantify the relative contribution of each vital rate to the population growth by a specific 
Table 6. Mean annual transition probability matrices.

\begin{tabular}{|c|c|c|c|c|c|c|c|c|}
\hline \multicolumn{9}{|c|}{ A. Agariciids } \\
\hline & SC1 & SC2 & SC3 & SC4 & SC5 & SC6 & SC7 & SC8 \\
\hline $\mathrm{SC} 1$ & 0.177 & 0.066 & 0.007 & 0.006 & 0 & 0 & 0 & 0 \\
\hline SC2 & 0.304 & 0.304 & 0.148 & 0.042 & 0.017 & 0.012 & 0 & 0.009 \\
\hline SC3 & 0.104 & 0.253 & 0.352 & 0.188 & 0.111 & 0.028 & 0.036 & 0.029 \\
\hline SC4 & 0.005 & 0.059 & 0.230 & 0.241 & 0.214 & 0.103 & 0.035 & 0.015 \\
\hline SC5 & 0.011 & 0.028 & 0.070 & 0.233 & 0.241 & 0.155 & 0.238 & 0.025 \\
\hline SC6 & 0 & 0.004 & 0.024 & 0.113 & 0.222 & 0.167 & 0.116 & 0.053 \\
\hline SC7 & 0 & 0.002 & 0.009 & 0.076 & 0.079 & 0.276 & 0.259 & 0.109 \\
\hline SC8 & 0 & 0 & 0.012 & 0.031 & 0.062 & 0.165 & 0.316 & 0.752 \\
\hline$s$ & 0.601 & 0.716 & 0.852 & 0.930 & 0.946 & 0.906 & 1.000 & 0.992 \\
\hline$d$ & 0.399 & 0.284 & 0.148 & 0.070 & 0.054 & 0.094 & 0 & 0.008 \\
\hline \multicolumn{9}{|c|}{ B. Porites asteroides } \\
\hline & $\mathrm{SC} 1$ & SC2 & SC3 & SC4 & SC5 & SC6 & SC7 & SC8 \\
\hline SC1 & 0.320 & 0.077 & 0.007 & 0 & 0 & 0 & 0 & 0 \\
\hline SC2 & 0.264 & 0.431 & 0.107 & 0.010 & 0 & 0.066 & 0 & 0 \\
\hline SC3 & 0.065 & 0.242 & 0.543 & 0.291 & 0.121 & 0 & 0 & 0 \\
\hline SC4 & 0 & 0.027 & 0.179 & 0.335 & 0.118 & 0.124 & 0.050 & 0 \\
\hline SC5 & 0 & 0.003 & 0.055 & 0.179 & 0.384 & 0.224 & 0.050 & 0 \\
\hline SC6 & 0 & 0 & 0.003 & 0.087 & 0.159 & 0.067 & 0.200 & 0.033 \\
\hline SC7 & 0 & 0 & 0 & 0.074 & 0.080 & 0.195 & 0.200 & 0.150 \\
\hline SC8 & 0 & 0 & 0 & 0 & 0.040 & 0.324 & 0.500 & 0.617 \\
\hline$s$ & 0.649 & 0.780 & 0.894 & 0.976 & 0.902 & 1.000 & 1.000 & 0.800 \\
\hline$d$ & 0.351 & 0.220 & 0.106 & 0.023 & 0.098 & 0 & 0 & 0.200 \\
\hline \multicolumn{9}{|c|}{ C. All 23 taxa pooled together } \\
\hline & $\mathrm{SC} 1$ & SC2 & SC3 & SC4 & SC5 & SC6 & SC7 & SC8 \\
\hline SC1 & 0.344 & 0.096 & 0.014 & 0.002 & 0 & 0 & 0 & 0 \\
\hline SC2 & 0.158 & 0.372 & 0.127 & 0.030 & 0.011 & 0.023 & 0 & 0.002 \\
\hline SC3 & 0.029 & 0.213 & 0.392 & 0.212 & 0.082 & 0.029 & 0.016 & 0.006 \\
\hline $\mathrm{SC} 4$ & 0.002 & 0.036 & 0.213 & 0.291 & 0.198 & 0.117 & 0.048 & 0.008 \\
\hline SC5 & 0.001 & 0.009 & 0.080 & 0.226 & 0.257 & 0.135 & 0.108 & 0.010 \\
\hline SC6 & 0 & 0.002 & 0.022 & 0.100 & 0.187 & 0.181 & 0.151 & 0.019 \\
\hline SC7 & 0 & 0.001 & 0.009 & 0.067 & 0.142 & 0.281 & 0.204 & 0.080 \\
\hline SC8 & 0 & 0.001 & 0.007 & 0.021 & 0.071 & 0.199 & 0.468 & 0.864 \\
\hline$s$ & 0.534 & 0.730 & 0.864 & 0.949 & 0.948 & 0.965 & 0.995 & 0.989 \\
\hline$d$ & 0.466 & 0.270 & 0.136 & 0.051 & 0.052 & 0.035 & 0.005 & 0.012 \\
\hline
\end{tabular}

Mean probabilities from 2010-11, 2011-12, 2012-13, 2013-14, and 2014-15 annual comparisons. Columns depict starting state, rows depict ending fate according to Equation (1). $s$ = probability of colony survival (the sum of the transition probabilities in the respective column); $d=$ probability of whole colony death $(1-s)$. 
Table 7. Stable size class distributions, dominant eigenvalues, and damping ratios.

\begin{tabular}{cccc}
\hline & AGAR & PAST & ALL \\
\hline Stable SC Distributions & & & \\
SC1 & $12 \%$ & $1 \%$ & $1 \%$ \\
SC2 & $21 \%$ & $6 \%$ & $2 \%$ \\
SC3 & $25 \%$ & $18 \%$ & $5 \%$ \\
SC 4 & $13 \%$ & $11 \%$ & $6 \%$ \\
SC5 & $11 \%$ & $11 \%$ & $6 \%$ \\
SC6 & $6 \%$ & $8 \%$ & $6 \%$ \\
SC7 & $4 \%$ & $12 \%$ & $10 \%$ \\
SC 8 & $8 \%$ & $33 \%$ & $64 \%$ \\
$\lambda$ Dominant Eigenvalue & & & \\
$\lambda$ & 0.94 & 0.88 & 0.97 \\
Damping Ratio & & & \\
$\Lambda_{1 /}\left|\lambda_{2}\right|$ & 1.36 & 1.16 & 1.29 \\
\hline
\end{tabular}

Stable size class distributions and eigenvalues from mean annual transition probability matrices.

amount and by a specific proportion, respectively [6] [7]. Sensitivity and elasticity matrices, displayed graphically as surface plots (Figure 6), indicated that the dominate eigenvalues, $\lambda$, are most affected by changes in the upper right corners of the matrices which correspond to the stability of SC8, the partial mortality of SC8 colonies into smaller size classes, and the growth of SC7 into SC8 colonies.

\subsection{Population Projections}

The mean annual size class transition probability matrices were used to project populations within the monitoring stations through 2040 (Figure 7), the year by which the reefs around Little Cayman has been projected to experience annual severe coral bleaching [13] [14]. These projections are idealized, best case scenarios which assume no environmental disturbances or changes in the vital rates (i.e. size class transition probabilities, recruitment rates).

1) The number of $P$. asteroides colonies is projected to decline by $23 \%$ over the 13 years following this study until a stable population distribution is reached. The largest changes in the population structure are projected to be a $16 \%$ decline in SC1, a 10\% decline in SC2, and a $10 \%$ increase in SC8. The mid-sized colonies (SC3-SC7) are projected to increase by $\leq 4 \%$ each. The trend toward larger colonies is projected to nearly balance with the decline in the number of colonies, resulting in a $\leq 1 \%$ increase in the total area cover of $P$. asteroides in 2040 compared to 2015 (assuming the mean surface area of SC8 corals remains unchanged within this timeframe).

2) The number of agariciid colonies is projected to decline by $11 \%$ for six years, followed by a gradual 13 -year recovery before stabilizing at $93 \%$ of 

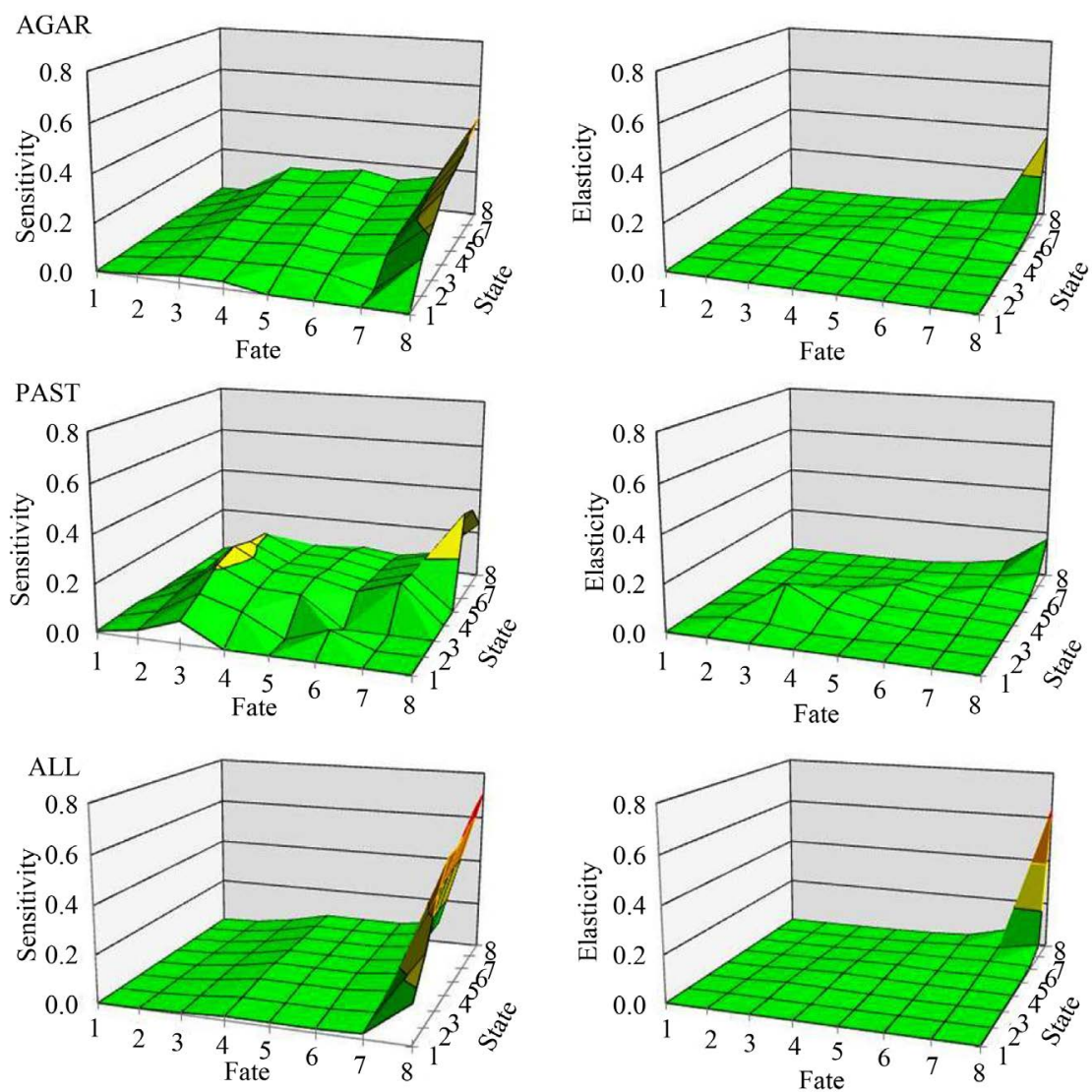

Figure 6. Sensitivity and Elasticity Surface Plots. Fate and State axes represent transitions between size classes. Vertical axes represent the sensitivity and elasticity of the respective population growth rates, $\lambda$, to perturbation analyses. Color coding: $0.0-0.2=$ green; 0.2 $0.4=$ yellow; $0.4-0.6=$ orange; $0.6-0.8=$ red.

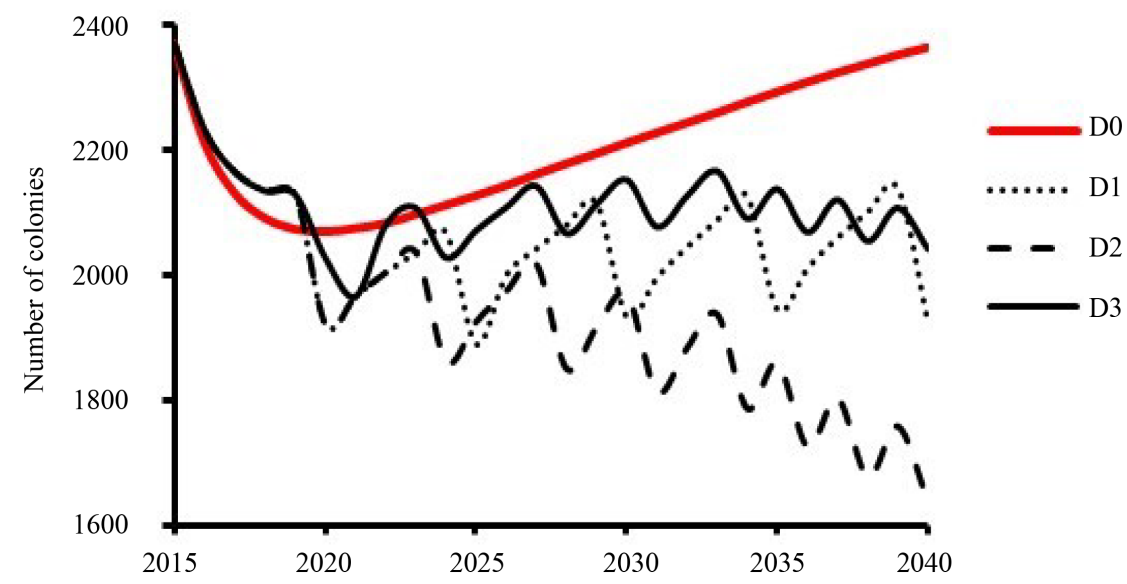

Figure 7. Projected Impacts of Mild Disturbance Scenarios on Hard Coral Population. Idealized projections assume no changes in size class transition probabilities or recruitment rates following each disturbance. D0 = Disturbance Scenario 0 (zero disturbances); D1 = Disturbance scenario \#1 (10\% mortality every 5 years); D2 = Disturbance scenario \#2 (10\% mortality with increasing disturbance frequency from every 5 years to every 2 years); and D3 = Disturbance scenario \#3 (5\% mortality with same increasing frequency as D2). 
the starting population. The largest changes are projected to be $6 \%-9 \%$ decreases in SC1-SC3 and a 14\% increase in SC8. The total area cover of agariciids is projected to be $2 \%$ higher in 2040 than in 2015.

3) The number of hard corals (all taxa pooled together) is projected to decline by $13 \%$ for six years, and to require 19 years to fully recover to the population size recorded at the beginning of this study in 2010. The largest change is projected to be a $22 \%$ increase in SC8 colonies (Figure 2). Insufficient sample sizes and data were available (i.e. mean surface areas of SC8 colonies for each of the 18 common and rare taxa groups) to project the impact this change may have on the total area cover.

Projections were recalculated for the hardcoral population within the monitoring stations under three mild disturbance scenarios (Figure 7). Each scenario assumes no change in size class transition probabilities or recruitment rates after each disturbance. An interval of five years between disturbance events was chosen to start each scenario because this is the mean timeframe between the seven most recent bleaching events observed in Little Cayman since 1985 which resulted in $\geq 5 \%$ mortality [3] [4] [15] [16] [17]. In the months following the 2015 data collection for this study, Little Cayman experienced an elevated temperature anomaly that resulted in $>80 \%$ paling and bleaching in many taxa including S. siderea [18], agariciids, Orbicella annularis, O. faveolata, O. franksi, Porites furcata, and $S$. radians (unpublished data). However, recovery was widespread with only an estimated $5 \%-10 \%$ mortality (personal observations). The disturbance scenarios were, therefore, based on the most recent mortality event in Little Cayman. Scenario \#1 is based on $10 \%$ mortality every five years. Scenarios \#2 (10\% mortality) and \#3 (5\% mortality) have the following disturbance frequencies: one five-year interval, two four-year intervals, two three-year intervals, and two two-year intervals. Scenarios \#1 and \#3 give resilient populations sufficient time to recover from the mild disturbances but not enough to recover from the downward trends that are projected to continue during non-disturbance years. Scenario \#2 results in a steadily declining population which is projected to drop $30 \%$ by 2040 .

\section{Discussion}

Agariciids, Porites asteroides, and Siderastrea radians have replaced acroporids as the predominant massive corals on the shallow reefs around Little Cayman. These resilient and resistant corals have characteristics of "long-term winners" [19]: the agariciids rapidly regrow following disturbances; $P$. asteroides are thermally tolerant and persistent; and $S$. radians are prolific recruiters.

Agariciids are the most dynamic of the hard coral taxa: recruit survival is relatively high compared to other taxa and colonies are capable of transitioning as many as six size classes up or down in a single year. Those colonies that reach SC4-SC8 $\left(\geq 30 \mathrm{~cm}^{2}\right)$ have a $\geq 90 \%$ chance of survival to the next year. These life history traits are critical because agariciids around Little Cayman are susceptible 
to bleaching [3] [4] and were observed to be among the early indicators of reef stress during the elevated seawater temperatures which followed the end of this study in 2015. These corals have the capacity to recover quickly after a disturbance compared to other taxa. Yet, despite this resiliency, the agariciid population is slowly declining because whole colony mortality currently exceeds recruitment.

Porites asteroides are characterized as persistent because they are slow to change: two-thirds of the colonies either remain in the same size class or transition (grow/shrink) only one size class each year. Previous studies in St. John, U.S. Virgin Islands recorded similar size class transition probabilities among this species [20]. Mid-sized $P$. asteroides colonies $\left(12-150 \mathrm{~cm}^{2}\right)$ have a $\geq 90 \%$ chance of survival to the next year during non-disturbance environmental conditions. Vital rates indicate that the slight decrease in the number of colonies is balanced by the growth of the surviving colonies such that live area cover is relatively stable.

Siderastrea radians are the most prolific recruiters during this study; however, the high recruit settlement is accompanied by the lowest recruit survival among the taxa. The recruitment pulse that occurred in 2011 did not result in a significant increase in coral area cover. $S$. radians colonies remain small, rarely exceeding SC2 $\left(12 \mathrm{~cm}^{2}\right)$ and together contribute only $1 \%$ of the live area cover.

The ecological succession of these taxa, along with $S$. siderea, following the loss of acroporids has been observed on reefs throughout the Caribbean [21]. The results of this study indicate that the ecological succession in Little Cayman may have reached its peak and 25\% live coral cover [1] [4] may be the new best-case scenario. The overall hard coral population is projected to gradually decline even during non-disturbance environmental conditions (i.e. conditions similar to those which occurred in 2010-2015). Similar downward population trends have also been projected for other Caribbean massive coral assemblages such as $P$. asteroides, Diploria strigosa, and Orbicella annularis in the U.S. Virgin Islands [21] [22] as well as $O$. annularis and Agaricia agaricites in Jamaica [23].

Coral communities have been shown to require 10 - 30 years to recover after a significant mortality event (e.g. [24] [25] [26] [27]. However, the frequency, intensity, and duration of disturbances that impact coral reefs are predicted to increase as a result of climate change [28]-[33]. Temperature-induced bleaching disturbances already occur on average every five years in Little Cayman and are expected to become an annual event around 2040 [13] [14]. Such regularity may not allow sufficient time for full recovery to pre-disturbance community structures.

\section{Conclusions}

Little information pertaining to hard coral vital rates in the Cayman Islands have been published to date. The population dynamics reported in this study may be 
used as baseline comparisons when conducting reef health surveys, when reporting the effects of disturbances on coral communities, or when developing predictive ecological models. With declining populations during non-disturbance conditions, Little Cayman's reefs are at risk of severe degradation should a large proportion of the SC8 colonies become compromised due to natural or anthropogenic stresses. Current recruitment levels cannot replace losses associated with minor disturbance events (e.g. 5\% - 10\% mortality).

The fate of hard corals on Little Cayman's reefs was determined to be heavily dependent on the health and transitions of agariciid colonies. Conservation strategies that currently focus on Acropora cervicornis and A. palmata restoration e.g. [34] [35] should be expanded to include agariciids, which were previously considered "weeds". Improvements to agariciid transition probabilities that increase survival and progression toward the stable size class distribution (e.g. recruit settlement, recruit and SC1-3 survival, growth towards SC8 colony sizes) may slow or reverse the projected population declines while ongoing efforts to restore the keystone coral populations are underway.

\section{Acknowledgements}

The authors thank the Cayman Islands Department of Environment for issuing the permit to install the monitoring station markers. The Central Caribbean Marine Institute (CCMI) allowed the authors to collect underwater images during its undergraduate field programs and annual monitoring dive trips. The National Science Foundation provided partial funding for travel to/from Little Cayman and field support during the 2014-2016 Research Experience for Undergraduates (Grant OCE-1358600).

\section{References}

[1] Jackson, J.B.C, Donovan, M.K., Cramer, K.L. and Lam, W. (2014) Status and Trends of Caribbean Coral Reefs: 1970-2012. Global Coral Reef Monitoring Network, IUCN, Gland, Switzerland.

[2] Dromard, C.R., McCoy, C.M.R. and Turner, J.R. (2010) Measuring the Performance of Marine Protected Areas: the Case of Little Cayman and Cayman Brac, Cayman Islands. Proceedings of the $63 \mathrm{rd}$ Gulf and Caribbean Fisheries Institute, San Juan, Puerto Rico, 246-253.

[3] van Hooidonk, R.J., Manzello, D.P., Moye, J., Brandt, M.E., Hendee, J.C., McCoy, C. and Manfrino, C. (2012) Coral Bleaching at Little Cayman, Cayman Islands 2009. Estuarine, Coastal and Shelf Science, 106, 80-84. https://doi.org/10.1016/j.ecss.2012.04.021

[4] Manfrino, C., Jacoby, C.A., Camp, E. and Frazer, T.K. (2013) A Positive Trajectory for Corals at Little Cayman Island. PLoS ONE, 8, e75432. https://doi.org/10.1371/journal.pone.0075432

[5] Kohler, K.E. and Gill, S.M. (2006) Coral Point Count with Excel Extensions (CPCe): A Visual Basic Program for the Determination of Coral and Substrate Coverage Using Random Point Count Methodology. Computers and Geosciences, 32, 1259-1269. https://doi.org/10.1016/j.cageo.2005.11.009 
[6] Caswell, H. (2001) Matrix Population Models: Construction, Analysis, and Interpretation. Sinauer Associates, Inc., Sunderland, MA.

[7] Owen-Smith, N. (2007) Introduction to Modeling in Wildlife and Resource Conservation. Blackwell Publishing, Malden, MA.

[8] Hughes, T.P. and Jackson, J. (1985) Population Dynamics and Life Histories of Foliaceous Corals. Ecological Monographs, 55, 141-166. https://doi.org/10.2307/1942555

[9] Babcock, R.C. (1991) Comparative Demography of Three Species of Scleractinian Corals Using Age- and Size-Dependent Classifications. Ecological Monographs, 61, 225-244. https://doi.org/10.2307/2937107

[10] Hughes, T.P. (1984) Population Dynamics Based on Individual Size Rather than Age: A General Model with a Reef Coral Example. The American Naturalist, 123, 778-795. https://doi.org/10.1086/284239

[11] Hood, G.M. (2010) PopTools Version 3.2.5. http://www.poptools.org

[12] Westerberg, L. and Wennergren, U. (2007) Matrix Models: A Tool for Landscape Management? In: Antonello, S.D., Ed., Frontiers in Ecology Research, Nova Science Publishers, New York, 141-163.

[13] van Hooidonk, R., Maynard, J., Tamelander, J., Gove, J., Ahmadia, G., Raymundo, L., Williams, G., Heron, S.F. and Planes, S. (2016) Local-Scale Projections of Coral Reef Futures and Implications of the Paris Agreement. Scientific Reports, 6, Article No. 39666. https://www.nature.com/articles/srep39666 https://doi.org/10.1038/srep39666

[14] van Hooidonk, R., Maynard, J., Tamelander, J., Gove, J., Ahmadia, G., Raymundo, L., Williams, G., Heron, S.F., Tracey, D., Parker, B. and Planes, S. (2017) Coral Bleaching Futures-Downscaled Projections of Bleaching Conditions for the World's Coral Reefs, Implications of Climate Policy and Management Responses. United Nations Environment Programme, Nairobi, 69 p.

[15] Ghiold, J. and Smith, S. (1990) Bleaching and Recovery of Deep-Water, Reef Dwelling Invertebrates in the Cayman Islands, British West Indies. Caribbean Journal of Science, 26, 52-61.

[16] Coehlo, V. and Manfrino, C. (2007) Coral Community Decline at a Remote Caribbean Island: Marine No-Take Reserves Are Not Enough. Aquatic Conservation: Marine and Freshwater Ecosystems, 17, 666-685. https://doi.org/10.1002/aqc.822

[17] Eakin, C., Morgan, J., Heron, S., Smith, T., Liu, G., et al. (2010) Caribbean Corals in Crisis: Record Thermal Stress, Bleaching, and Mortality in 2005. PLOS ONE, 5, e13969. https://doi.org/10.1371/journal.pone.0013969

[18] Banks, S. and Foster, K. (2017) Baseline Levels of Siderastrea siderea under Normal Environmental Conditions in Little Cayman. Open Journal of Marine Science, 7, 142-154. https://doi.org/10.4236/ojms.2017.71011

[19] Van Woesik, R., Sakai, K., Ganase, A. and Loya, Y. (2011) Revisiting the Winners and the Losers a Decade after Coral Bleaching. Marine Ecology Progress Series, 434, 67-76. https://doi.org/10.3354/meps09203

[20] Edmunds, P.J. (2010) Population Biology of Porites asteroides and Diploria strigosa on a Shallow Caribbean Reef. Marine Ecology Progress Series, 418, 87-104. https://doi.org/10.3354/meps08823

[21] Perry, C.T., Steneck, R.S., Murphy, G.N., Kench, P.S., Edinger, E.N., Smithers, S.G. and Mumby, P.J. (2015) Regional-Scale Dominance of Non-Framework Coral on Caribbean Reefs Affects Carbonate Production and Future Reef Growth. Global Change Biology, 21, 1153-1164. https://doi.org/10.1111/gcb.12792 
[22] Edmunds, P.J. (2015) A Quarter-Century Demographic Analysis of the Caribbean Coral, Orbicella annularis, and Projections of Population Size over the Next Century. Limnology and Oceanography, 60, 840-855. https://doi.org/10.1002/lno.10075

[23] Hughes, T.P. and Tanner, J.E. (2000) Recruitment Failure, Life Histories, and Long-Term Decline of Caribbean Corals. Ecology, 81, 2250-2263. https://doi.org/10.1890/0012-9658(2000)081[2250:RFLHAL]2.0.CO;2

[24] Connell, J.H. (1997) Disturbance and Recovery of Coral Assemblages. Coral Reefs, 19, S101-S113. https://doi.org/10.1007/s003380050246

[25] Ninio, R., Meekan, M., Done, T. and Sweatman, H. (2000) Temporal Patterns in Coral Assemblages on the Great Barrier Reef from Local to Large Spatial Scales. Marine Ecology Progress Series, 194, 65-74. https://doi.org/10.3354/meps194065

[26] Bruno, J.F. and Selig, E.R. (2007) Regional Decline of Coral Cover in the Indo-Pacific: Timing, Extent and Subregional Comparisons. PLoS ONE, 2, e711. https://doi.org/10.1371/journal.pone.0000711

[27] Foster K.A., Foster, G. and Al-Harthi, S. (2013) Coral Assemblages in the Southeastern Arabian Gulf (Qatar and Abu Dhabi, UAE): Various Stages of Acropora Eecovery a Decade after Recurrent Elevated Temperature Anomalies. Open Journal of Marine Science, 3, 28-29. https://doi.org/10.4236/ojms.2013.32A004

[28] Hoegh-Guldberg, O. (1999) Climate Change, Coral Bleaching and the Future of the World's Coral Reefs. Marine and Freshwater Research, 50, 839-866. https://doi.org/10.1071/MF99078

[29] Emanual, K. (2005) Increasing Destructiveness of Tropical Cyclones over the Past 30 Years. Nature, 436, 686-688. https://doi.org/10.1038/nature03906

[30] Webster, P., Holland, G., Curry, J.A. and Chang, H.-R. (2005) Changes in Tropical Cyclone Number, Duration, and Intensity in a Warming Environment. Science, 309, 1844-1847. https://doi.org/10.1126/science.1116448

[31] Riegl, B.M. and Purkis, S.J. (2009) Model of Coral Population Response to Accelerated Bleaching and Mass Mortality in a Changing Climate. Ecological Modeling, 220, 192-208. https://doi.org/10.1016/j.ecolmodel.2008.09.022

[32] Fabina, N.S., Baskett, M.L. and Gross, K. (2015) The Differential Effects of Increasing Frequency and Magnitude of Extreme Events on Coral Populations. Ecological Applications, 25, 1534-1535. https://doi.org/10.1890/14-0273.1

[33] Randall, C.J. and van Woesik, R. (2017) Some Coral Diseases Track Climate Oscillations in the Caribbean. Scientific Reports, 7, Article No. 5719.

https://www.nature.com/articles/s41598-017-05763-6 https://doi.org/10.1038/s41598-017-05763-6

[34] Lohr, K.E., Bejarano, S., Lirman, D., Schopmeyer, S. and Manfrino, C. (2015) Optimizing the Productivity of a Coral Nursery Focused on Staghorn Coral Acropora cervicornis. Endangered Species Research, 27, 243-250. https://doi.org/10.3354/esr00667

[35] Lohr, K.E., Cook McNab, A.A, Manfrino, C. and Patterson, J.T. (2017) Assessment of Wild and Restored Staghorn Coral Acropora cervicornis across Three Reef Zones in the Cayman Islands. Regional Studies in Marine Science, 9, 1-8.

https://doi.org/10.1016/j.rsma.2016.11.003 